\title{
Anaerobic Growth as a Factor Influencing Radiosensitivity
}

\author{
BY BRENDA HODGKINS AND TIKVAH ALPER \\ Medical Research Council, Experimental Radiopathology Research Unit, \\ Hammersmith Hospital, Ducane Road, London, W.12
}

(Received 7 June 1962)

\begin{abstract}
SUMMARY
Damage to living organisms by $\mathrm{X}$-rays (but not by ultraviolet radiation) is generally enhanced by the presence of oxygen, which probably acts at a physico-chemical level. Sensitivity to both u.v.- and X-radiation may also be related to the presence of oxygen acting as a metabolic factor during the growth of micro-organisms before or after irradiation.

Anaerobic growth of Escherichia coli strain B after u.v. or X-rays decreased lethal damage, the treatment being most effective afteru.v.-and more effective after $\mathbf{X}$-rays delivered in the absence than in the presence of oxygen. In this respect anaerobic growth corresponded with a variety of inhibitory conditions after irradiation, and rescue by anaerobic growth was about as effective as chloramphenicol treatment; but when these two treatments were used together, the effectiveness of each was decreased.

The effects of u.v.- and X-radiation on Escherichia coli $\mathbf{B} / \mathbf{r}$ were unchanged by anaerobic growth after irradiation; but anaerobic growth beforehand resulted in survival curves which were much more sigmoid in shape than those pertaining to aerobically grown organisms. This tendency was noted with all strains tested. Anaerobic growth before Xirradiation did not alter the effect of oxygen during irradiation.
\end{abstract}

\section{INTRODUCTION}

Various lines of evidence support the view that oxygen, which enhances the effectiveness of ionizing radiation when present during irradiation, acts physicochemically, rather than by affecting metabolism. With some strains of microorganism, effects of radiation on colony-forming ability may be greatly modified by changing the conditions of growth, both before and after irradiation. Modificacation of this type is clearly related to effects on the biochemical processes which lead to cell division, and the presence or absence of oxygen during growth of the organisms may affect the end result. The mechanisms of this modifying action of oxygen are easily distinguishable from those involved in the part which oxygen plays by intervening in the 'metionic reaction', i.e. the chemical reaction immediately following an ionizing event (Alper, 1956).

It was reported by Alper \& Gillies (1960 a) that Escherichia coli strain B organisms when kept anaerobic after X- or u.v.- irradiation behaved like those to which other metabolic inhibitors were applied in that their colony-forming ability was much less affected than when they were grown in optimal conditions. Effects of anaerobic growth on this strain, and on its mutant $E$. coli $\mathrm{B} / \mathrm{r}$, have now been studied in greater detail. 


\section{METHODS}

Organisms. The organisms used in most of the experiments were Escherichia coli strains B and B/r, as used in previous work in this laboratory. A few comparative experiments were done with $E$. coli strains $\mathrm{K} 12(\lambda)$ and K12S, and with Salmonella typhimurium strain LT2.

Media. Oxoid blood-agar base was used as plating medium and for slopes. Oxoid nutrient broth no. 2 was used for broth cultures.

Preparation of organisms. Logarithmic-phase broth cultures were prepared by inoculating $0.2 \mathrm{ml}$. of an overnight broth culture into $5 \mathrm{ml}$. of fresh broth which was incubated for $90 \mathrm{~min}$. at $37^{\circ}$ with a moderate degree of aeration. A further dilution of this culture was made and aeration continued for $105 \mathrm{~min}$. The organisms were then centrifuged down and washed thrice in $0.067 \mathrm{M}$-phosphate buffer $(\mathrm{pH} 7 \cdot 0)$ and finally resuspended in this buffer.

The most convenient way of attaining maximally aerobic conditions was to grow the organisms on slopes for a short time. Anaerobically grown cultures were prepared by using slopes incubated in a McIntosh \& Fildes anaerobic jar so that the conditions of growth were the same in all but this one respect. All aerobically grown slope cultures were harvested after growth for 4-4.5 hr.; anaerobic cultures were usually grown for the same time, but in a few experiments they were left long enough to enter the stationary phase. The organisms were harvested by washing off in phosphate buffer, and were then washed twice more.

For ultraviolet- (u.v.-) irradiations the organisms were spread very thinly on cellophan carriers supported on $\mathbf{1 . 5} \%$ agar in distilled water, a techniquedescribed by Alper \& Gillies (1958). Suspensions of organisms were exposed to X-rays or to $8 \mathrm{MeV}$. electrons in specially designed vessels through which air or oxygen-free nitrogen was continuously bubbled (Alper, 1955).

Sources of radiation. (1) Ultraviolet-irradiations were carried out by using a waterjacketed Hanovia 'Germicidal' lamp, $90 \%$ of the energy being emitted at $2537 \AA$.; the dose rate was about $200 \mathrm{ergs} / \mathrm{mm} .^{2} / \mathrm{min}$. (2) For X-irradiations we used a Solus Schall $300 \mathrm{kV}$. constant potential X-ray unit, operating at $250 \mathrm{kV}$. and $10 \mathrm{~mA}$., with no added filtration. The dose rate in the vessels was 1050 rads./min. as determined by ferrous dosimetry. (3) In certain experiments we used the $8 \mathrm{MeV}$. linear accelerator of the M.R.C. Radiotherapeutic Research Unit (Batchelor, Bewley, Morrison \& Stevenson, 1959). The arrangements for irradiation were as described by Alper (1959).

Post-irradiation treatment. All nutrient agar plates were warmed to $37^{\circ}$ before transferring to the surface either samples of suspensions of organisms, or cellophan carriers.

Anaerobic conditions were obtained by using a McIntosh \& Fildes anaerobic jar, evacuating and refilling with pure hydrogen twice. The jar was maintained at $37^{\circ}$ throughout. After overnight incubation air was admitted and the incubation continued until the colonies were large enough to count (about $6 \mathrm{hr}$.) Since we wished to establish comparative rates of growth on plates, under aerobic and anaerobic conditions, the technique described by Alper \& Gillies (1960 a) was used. Organisms were seeded on to strips of cellophan supported on the surface of nutrient agar, removed after suitable intervals and shaken in MacCartney bottles containing phosphate buffer. Viable counts were made on the suspensions so obtained. 
Chloramphenicol treatment. In general we followed the methods described by Gillies \& Alper (1959). Cellophan carriers on which the organisms had been irradiated were laid on the surface of nutrient agar chloramphenicol and left overnight in aerobic or anaerobic conditions. The carriers were then transferred to normal nutrient agar and incubated until the colonies were large enough to count.

In one experiment air was admitted to chloramphenicol agar plates which had been incubated anaerobically overnight and the organisms incubated aerobically for a few hours on chloramphenicol agar, in case the anaerobiosis had prevented the chloramphenicol from exerting its full effect. The final result was not affected.

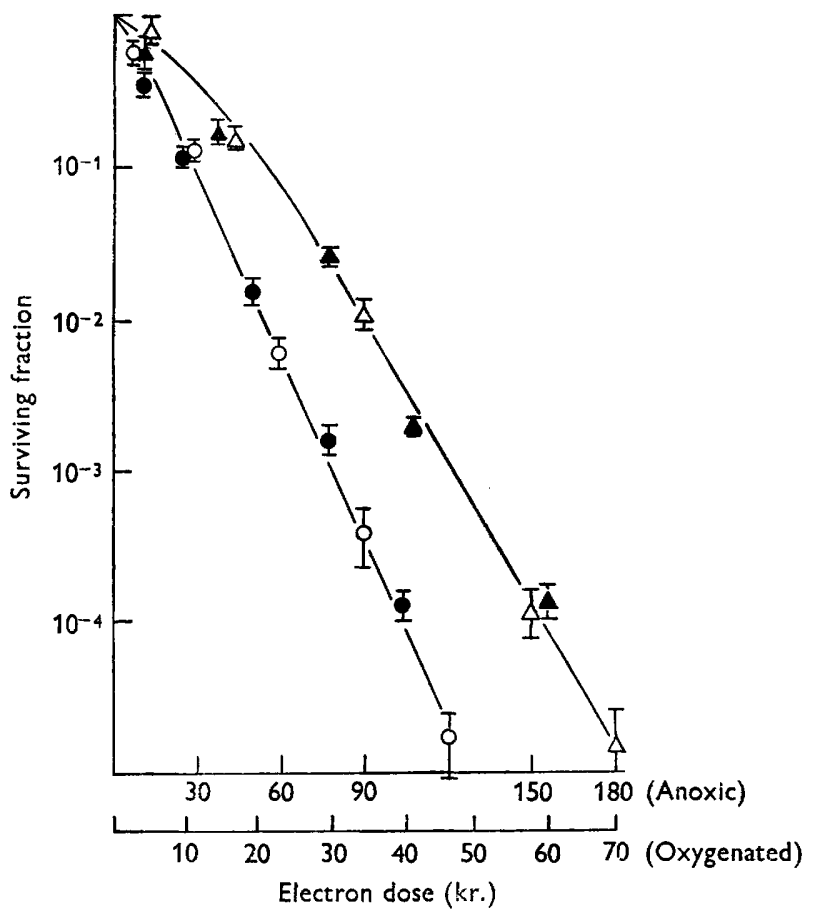

Fig. 1

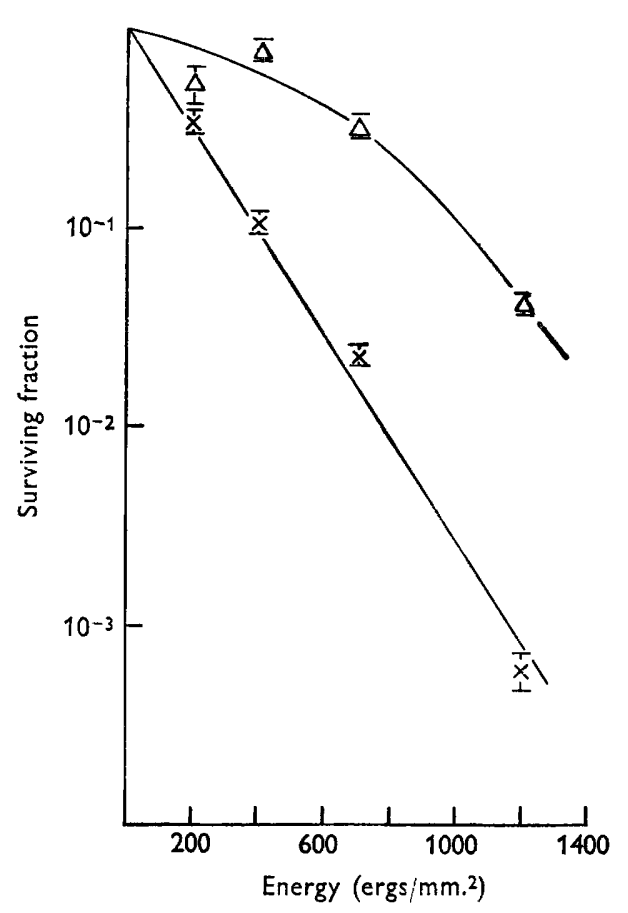

Fig. 2

Fig. 1. Survival of Escherichia coli strain B/r (harvested from a slope growing aerobically or anaerobically) after exposure in buffer solution to $\mathrm{X}$-rays under oxygenation or anoxia. Subsequent incubation aerobic. O, Grown aerobically, irradiated under oxygen; $O$, grown aerobically, irradiated under anoxia; $\Delta$, grown anaerobically, irradiated under oxygen; $\triangle$, grown anaerobically, irradiated under anoxia. Dosage scales have been selected so that survival curves for irradiations with and without oxygen are superimposed. $95 \%$ confidence limits on every point are indicated.

Fig. 2. Survival of Escherichia coli strain $B / \mathbf{r}$ (harvested from slopes grown aerobically or anaerobically) after exposure in buffer suspension to u.v.-radiation. Subsequent incubation aerobic. $x$, Grown aerobically before irradiation; $\Delta$, grown anaerobically before irradiation.

Chloramphenicol (5 $\mu \mathrm{g} . / \mathrm{ml}$.) was used in the experiments reported by Gillies \& Alper (1959); but in the present series we found a loss of viability in unirradiated organisms which had been left in contact with chloramphenicol $(5 \mu \mathrm{g} . / \mathrm{ml}$.) over- 
night. We cannot account for this increase in toxicity. Chloramphenicol at $1 \mu \mathrm{g} . / \mathrm{ml}$. had as much rescuing effect as was found previously with $5 \mu \mathrm{g} . / \mathrm{ml}$.; the lower concentration was used in the present experiments.

\section{RESULTS}

\section{Effect of anaerobiosis before harvesting organisms for irradiation}

Escherichia coli B/r. Young cultures of $E$. coli strain B/r grown on nutrient agar slopes yielded exponential or very nearly exponential survival curves, while those of the same age grown anaerobically yielded curves of equal slope, but with an initial

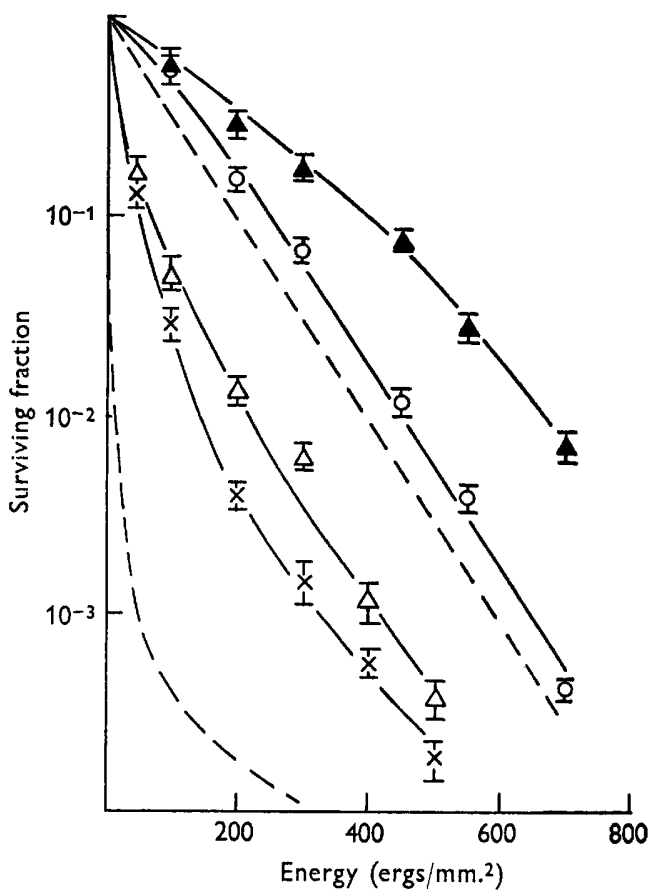

Fig. 3

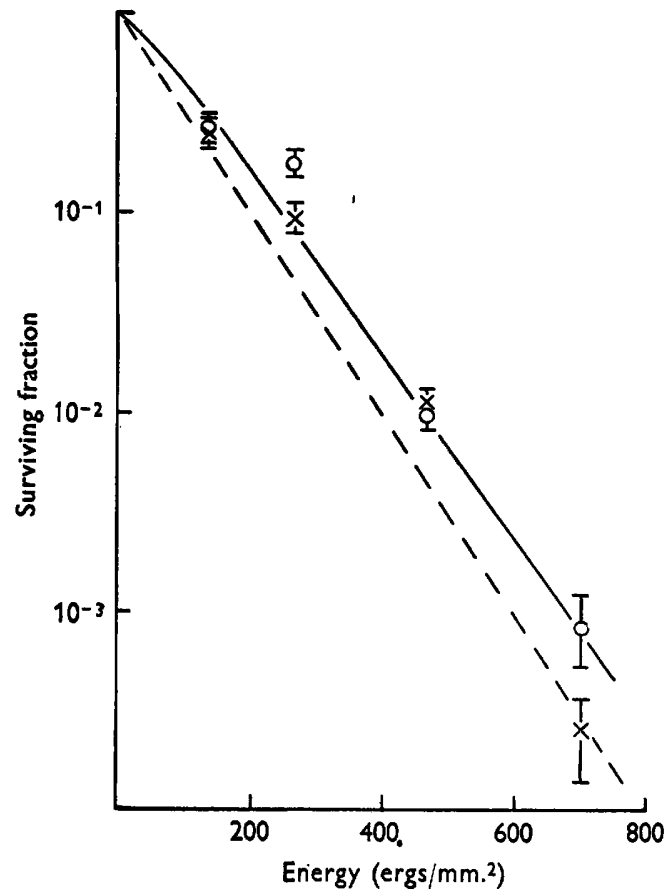

Fig. 4

Fig. 3. Survival of Escherichia coli strain B (harvested from slopes grown aerobically or anaerobically) after exposure on cellophan carriers to u.v.-radiation. $\times$, Grown aerobically, incubated aerobically; $O$, grown aerobically, incubated anaerobically; $\triangle$, grown anaerobically, incubated aerobically; $\Delta$, grown anaerobically, incubated anaerobically. The dashed curves indicate the survival of broth-grown logarithmic phase organisms grown aerobically or anaerobically after u.v.-irradiation (see Fig. 5).

Fig. 4. Survival of Escherichia coli strain B/r (logarithmic phase, broth-grown) after exposure on cellophan carriers to u.v.-radiation. $x$, Aerobic incubation; $O$, anaerobic incubation. The dashed curve indicates the survival of $E$. coli $\mathbf{B}$ grown under similar conditions and incubated anaerobically after u.v.-irradiation.

shoulder. Survival curves pertaining to anaerobically grown organisms had extrapolation numbers (Alper, Gillies \& Elkind, 1960) as high as 8 (Figs. 1, 2). The final slope of a survival curve with stationary-stage anaerobically grown organisms was only slightly less than that observed with logarithmic-phase organisms grown aerobically (see Fig. 1). Figure 1 shows that pairs of survival curves for the two 
populations, each irradiated in the presence and absence of oxygen, could be superimposed by using the same dose-multiplying factor $(2 \cdot 6$ in the conditions of the experiment illustrated). These results are in agreement with those reported by Hollaender, Stapleton \& Martin (1951), Howard-Flanders \& Alper (1957) and Sargent (1961). Figure 2 shows the difference in response to u.v.-irradiation of aerobically and anaerobically grown organisms of the same age.

Escherichia coli B. Organisms of this strain grown aerobically on nutrient agar slopes were not so sensitive to u.v.- or X-irradiation as were broth-grown organisms; those grown anaerobically were still less sensitive (Fig. 3).

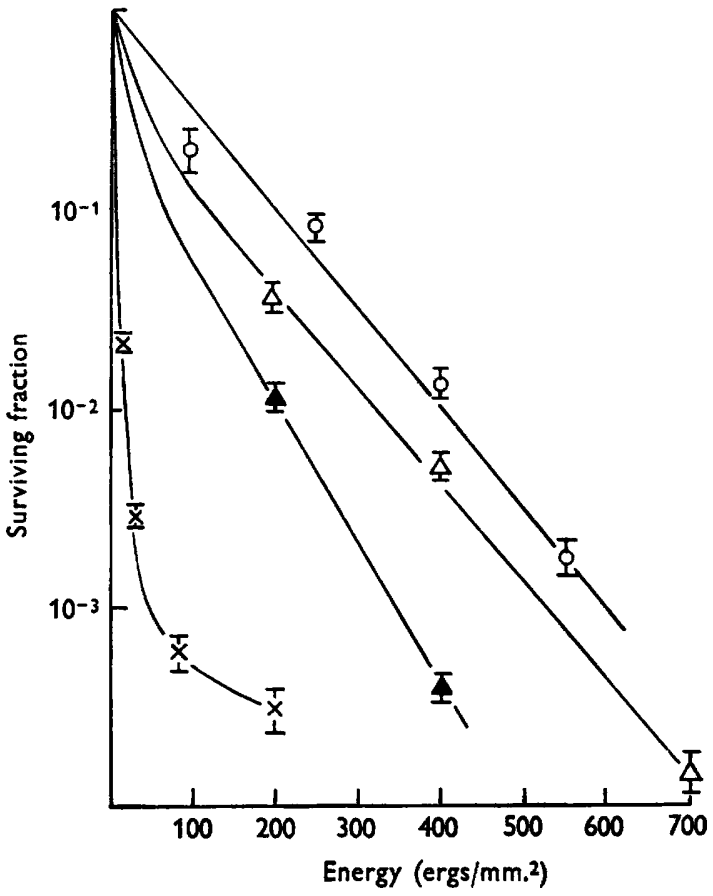

Fig. 5

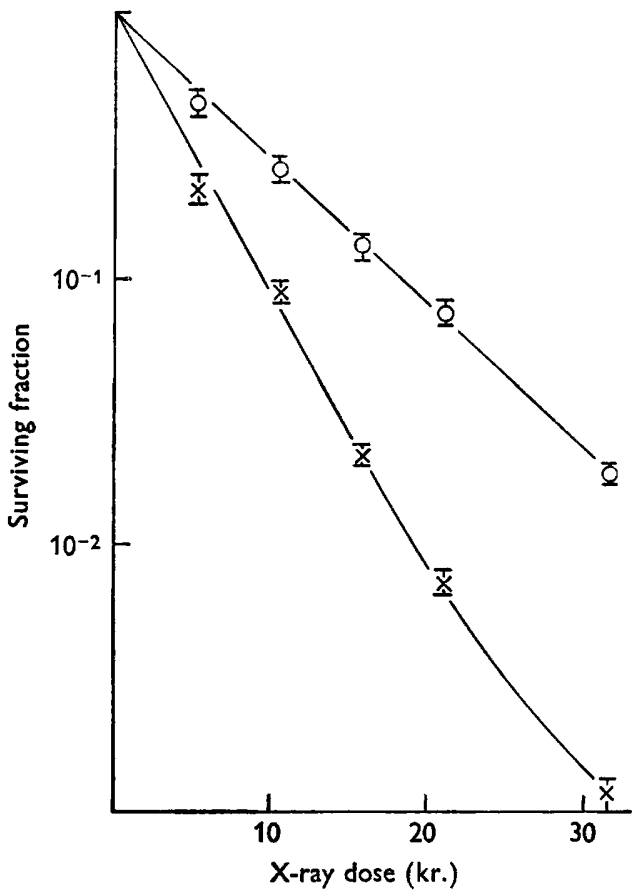

Fig. 6

Fig. 5. Survival of Escherichia coli B (logarithmic phase, broth-grown) after exposure on cellophan carriers to u.v.-radiation. $\times$, Aerobic incubation on Oxoid agar; $O$, anaerobic incubation on Oxoid agar; $\triangle$, aerobic incubation on Oxoid agar $+1 \mu \mathrm{g}$. chloramphenicol/ ml. followed by incubation on nutrient agar; $\Delta$, anaerobic incubation on Oxoid agar $+1 \mu \mathrm{g}$./chloramphenicol $/ \mathrm{ml}$., followed by incubation on nutrient agar.

Fig. 6. Survival of Escherichia coli strain B (logarithmic phase, broth-grown) after exposure in buffer suspension to $\mathrm{X}$-rays under anoxia. $\times$, Aerobic incubation after $\mathbf{X}$-irradiation; $\mathrm{O}$, anaerobic incubation after $\mathbf{X}$-irradiation.

Salmonella typhimurium LT2. This strain showed the same characteristics as did Escherichia coli $\mathrm{B} / \mathrm{r}$ : survival curves of anaerobically grown organisms showed an initial shoulder not seen with aerobically grown organisms.

\section{Anaerobiosis after irradiation}

Escherichia B/r. This organism showed no effect on the survival curve after u.v.(Fig. 4) or X-irradiation.

Escherichia B. Anaerobiosis was more effective in rescuing the organisms after 
u.v.-irradiation than after $X$-irradiation; irradiation was more effective when the organisms were $\mathrm{X}$-irradiated in the absence of oxygen than in its presence (Figs. 3 and 5-7). Up to the time of the first cell division, increasing duration of anaerobiosis permitted more $\mathrm{X}$ - or u.v.-irradiated organisms to give colonies; conversely, increasing periods of aerobic growth before anaerobiosis was started decreased the final colony counts until the subsequent imposition of anaerobiosis no longer affected the number of colonies formed. Figure 8 shows how the colony-forming ability varied when the organisms exposed to $200 \mathrm{ergs} / \mathrm{mm}^{2}$ of u.v.-radiation were transferred successively from an anaerobic to an aerobic environment and vice

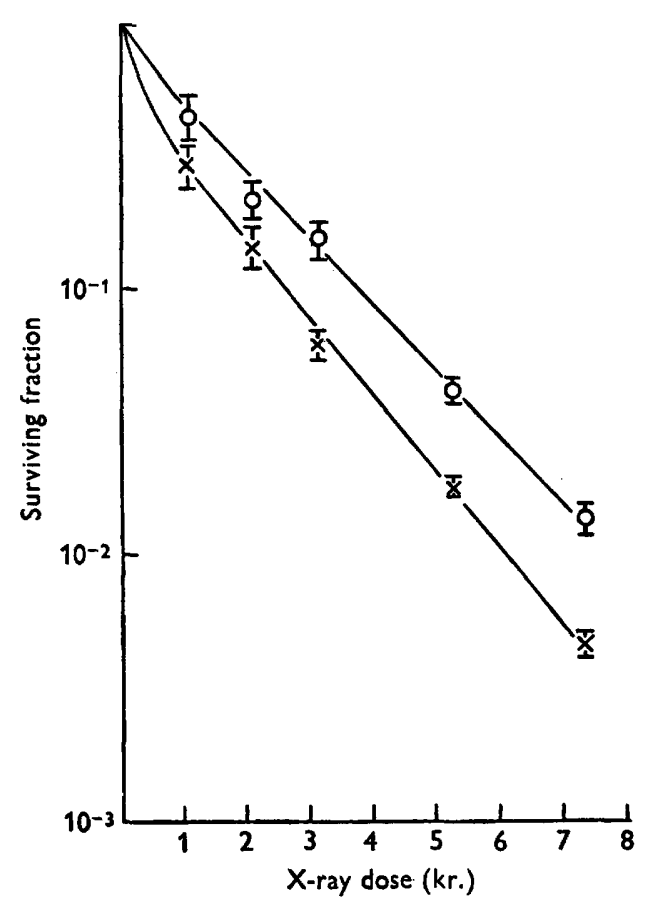

Fig. 7

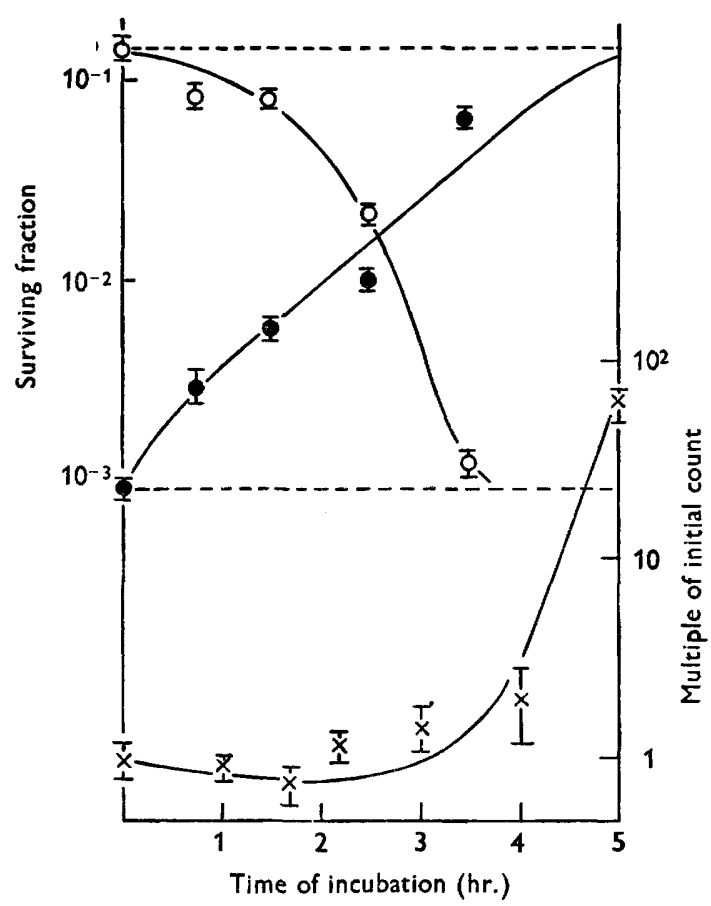

Fig. 8

Fig. 7. Survival of Escherichia coli strain B (logarithmic phase, broth-grown) after exposure in buffer suspension to $X$-rays under oxygen. $\times$, Aerobic incubation after $X$-irradiation; $O$, anaerobic incubation after $X$-irradiation.

Fig. 8. Survival and growth of Escherichia coli strain B (logarithmic phase, brothgrown) after exposure on cellophan carriers to $200 \mathrm{ergs} / \mathrm{mm} .^{2}$ u.v.-radiation. $\bigcirc$, Transferred from aerobic to anaerobic incubation at the time indicated on abscissa; 9 , transferred from anaerobic to aerobic incubation at the time indicated on abscissa; $\times$, viable counts, on u.v.-irradiated organisms growing aerobically, as multiple of initial count.

versa. The growth of the u.v.-irradiated organisms in aerobic conditions is shown. There was a similar dependence of survival on duration of aerobic or anaerobic growth after $\mathrm{X}$-irradiation. The dose of $\mathrm{X}$-rays required to give the same survival as $200 \mathrm{ergs} / \mathrm{mm} .^{2}$ u.v.-radiation imposed a shorter lag period, and the period within which change occurred was correspondingly shortened.

Other organisms. Anaerobiosis had no effect on Escherichia coli K $12(\lambda)$ or K $12 \mathrm{~S}$ after u.v.-irradiation. Salmonella typhimurium was affected like $E$. coli B, but to a lesser extent. 


\section{Effect of anaerobiosis on growth of Escherichia strains $B$} and $\boldsymbol{B} / \boldsymbol{r}$ (unirradiated)

Escherichia coli $\mathrm{B} / \mathrm{r}$ had the same lag phase and generation time whether grown aerobically or anaerobically. With $E$. coli $\mathrm{B}$, however, the lag phase and the generation time were increased (Fig. 9).

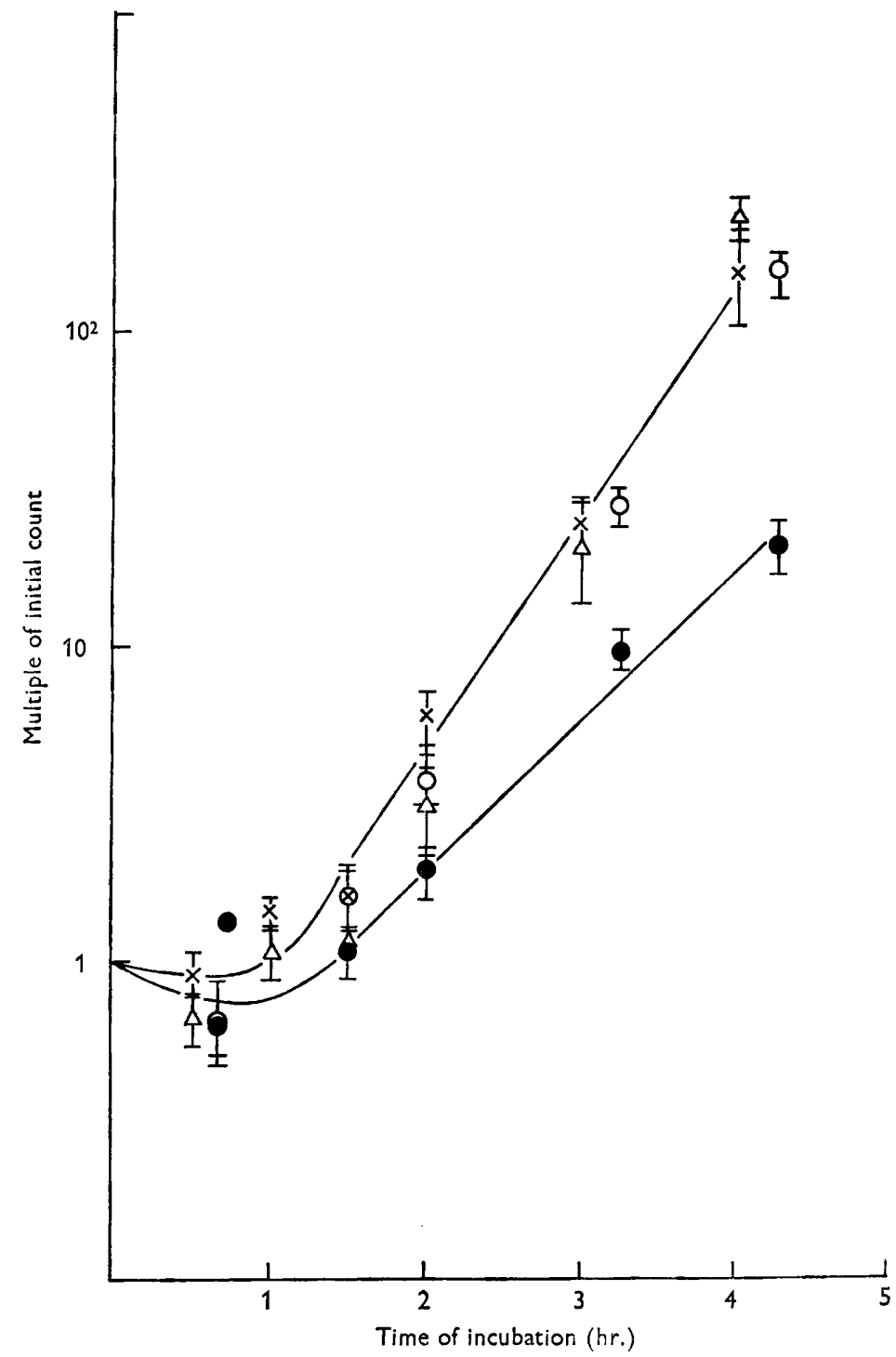

Fig. 9. Growth of non-irradiated Escherichia coli strains $B$ and $B / r$ (harvested in $\log$ arithmic phase from broth). $\times, E$. coli B growing aerobically;,$E$. coli B growing anaerobically; $\triangle, E$. coli $\mathrm{B} / \mathbf{r}$ growing aerobically; $O, E$. coli $\mathrm{B} / \mathrm{r}$ growing anaerobically. 


\section{Interaction of anaerobiosis with chloramphenicol treatment after irradiation}

Anaerobiosis as a mechanism for rescuing irradiated Escherichia coli B had slightly more effect than had chloramphenicol treatment when the latter was applied immediately after plating. As reported by Gillies \& Alper (1959), chloramphenicol treatment applied to u.v.-or X-irradiated organisms after short incubation on nutrient agar allowed more organisms to form colonies. When the counts were expressed as a dose-effect curve, the higher counts were seen to be due to the appearance of an initial shoulder on the survival curve. Chloramphenicol treatment in anaerobic conditions brought about much less rescue than anaerobic growth alone, or chloramphenicol treatment in aerobic conditions (Fig. 5).

\section{DISCUSSION}

Anaerobiosis as a rescuing treatment for u.v.-irradiated Escherichia coli $B$

It was suggested by Alper (1961, 1962) and by Gillies (1961) that the lethal effect of X- or u.v.-radiation on micro-organisms might occur as a result of damage in at least two sites, such that treatment after irradiation might act differentially on these. As observed with other treatments which rescue irradiated organisms of this strain of Escherichia coli there seems to be a minimum degree of damage which anaerobiosis is unable to affect; much the same dose-effect curves are seen when u.v.- or $\mathrm{X}$-irradiated $E$. coli $\mathrm{B}$ is treated by keeping the organisms anaerobic, by chloramphenicol, by specific inhibition of protein synthesis (Gillies, 1961) or simply by holding the organisms out of contact with nutrients (Roberts \& Aldous, 1949; Charles \& Zimmermann, 1956; Gillies, 1961). It seems probable that all such treatments enable the organisms to by-pass damage to a particular site, but the mechanism may very well be different with different treatments. Certainly the kinetics of rescue by chloramphenicol or by puromycin (Dr N. E. Gillies, personal communication) differ from those observed with other treatments we have used. As shown in Fig. 8, preliminary aerobic incubation decreased the effectiveness of subsequent anaerobiosis : similarly, a preliminary period of normal protein synthesis decreased the effectiveness of subsequent specific inhibition thereof (Gillies, 1961). The reverse is true of treatment by chloramphenicol and puromycin, their overall effectiveness being greater after a preliminary period of vigorous normal metabolism. But when chloramphenicol or puromycin was applied immediately, and for short periods, to u.v.- or X-irradiated organisms, particularly when these were in the logarithmic stage of growth, there was a decrease in the final colony count, not an increase (Gillies \& Alper, 1959; Dr N. E. Gillies, personal communication). Not only was there no additivity in the rescuing effect of chloramphenicol treatment and anaerobiosis, but these two treatments in fact counteracted each other (Fig. 5); this suggests that two different pathways of rescue from radiation damage may interfere with each other.

Anaerobiosis before u.v.- or $X$-irradiation. In all three strains tested, anaerobic growth decreased the effect of radiation on colony-forming ability. Anaerobic growth conferred on Escherichia coli $\mathrm{B} / \mathrm{r}$ and on Salmonella typhimurium the ability to absorb a dose of radiation before the killing became exponential with dose, while with $E$. coli B the slope of the survival curve was decreased. When $E$. coli B was 
grown anaerobically after irradiation, as well as before, the survival curve showed the same characteristics as did that pertaining to anaerobically grown $E$. coli strain B/r (Fig. 3). It was inferred by Alper \& Gillies $(1960 a, b)$ that the two strains of $E$. coli as normally grown (e.g. in nutrient broth) do not differ in the targets which they present to radiation, but rather in the effect which damage to these targets has on the biochemical pathways which lead to cell division. Like other treatments, anaerobiosis after u.v.-irradiation has the effect on $E$. coli $B$ of making its response to u.v.-radiation similar to that of its resistant mutant $B / r$, the growth curve of which is exactly the same in aerobic and in anaerobic conditions. It is noteworthy that anaerobic conditions, without affecting generation time, affect $E$. coli B/r so profoundly that the organisms acquire the ability to absorb a 'quasi-threshold dose' (Alper et al. 1962) which is about twice that required to produce an average of one lethal event per organism, once the curve has become exponential.

We are grateful to Dr N. E. Gillies for permission to quote some of his unpublished results.

\section{REFERENCES}

Alper, T. (1955). Bacteriophage as indicator in radiation chemistry. Radiat. Res. 2, 119.

Aluper, T. (1956). The modification of damage caused by primary ionization of biological targets. Radiat. Res. 5, 573.

AlPER, T. (1959). Variability of the oxygen effect observed with micro-organisms. Part I. Haploid yeast; single and budding cells. Int. J. rad. Biol. 1, 414.

ALPER, T. (1961). Variability of the oxygen effect observed with micro-organisms. Part II. Escherichia coli B. Int. J. rad. Biol. 3, 369.

Alper, T. (1962). Evidence for two resolvable sites of action of radiation on microorganisms. Abh. dtsch. Akad. Wiss., Berl. Klasse für Medizin, no. 1, p. 153.

Alper, T., Fowler, J. F., Morgan, R. L., Vonberg, D. D., Ellis, F. \& Oliver, R. (1962). The characterization of the 'Type C' survival curve. Brit. J. Radiol. 35, 722.

Alper, T. \& GiluIEs, N. E. (1958). Restoration of Escherichia coli strain B after irradiation: its dependence on suboptional growth conditions. J. gen. Microbiol. 18, 461.

AlPER, T. \& Gillies, N. E. (1960a). The relationship between growth and survival after irradiation of Escherichia coli strain B and two resistant mutants. J. gen. Microbiol. 22, 113.

AlPER, T. \& Gillies, N. E. (1960b). The influence of metabolic events immediately after irradiation on the difference in sensitivity between $\boldsymbol{E}$. coli B and $E$. coli B/r. 'Immediate and low level effects of ionizing radiations Conference, Venice June 1959.' Supplement to Int. J. rad. Biol. (1960), p. 305.

Alper, T., Gillies, N. E. \& Elkind, M. M. (1960). The sigmoid survival curve in radiobiology. Nature, Lond. 186, 1062.

Batchelor, A., Bewley, D. K., Morrison, R. \& Stevenson, J. A. (1959). Electron Therapy at $8 \mathrm{MeV}$. Brit. J. Radiol. 32, 332.

Charles, R. L. \& ZimmermanN, L. N. (1956). Dark reactivation in ultra-violet irradiated Escherichia coli. J. Bact. 71, 611.

Gillies, N. E. (1961). The use of auxotrophic mutants to study restoration in E. coli B after ultraviolet irradiation. Int. J. rad. Biol. $3,379$.

GILIIES, N. E. \& ALPER, T. (1959). Reduction in the lethal effects of radiations on Escherichia coli $B$ by treatment with chloramphenicol. Nature, Lond. 183, 287.

Hollaender, A., Stapleton, G. E. \& Martin, F. L. (1951). X-ray sensitivity of E. coli as modified by oxygen tension. Nature, Lond. 167, 103.

Howard-Flanders, P. \& Alper, T. (1957). The sensitivity of micro-organisms to irradiation under controlled gas conditions. Radiat. Res. 7, 518.

Roberts, R. B. \& Aldous, E. (1949). Recovery from ultraviolet irradiation in Escherichia coli. J. Bact. 57, 368.

SARGENT, T. (1961). Effects of $\mathrm{pH}$ and anoxia on the cell morphology and X-ray sensitivity of Escherichia coli. Radiat. Res. 14, 323. 